\title{
INTRINSIC MOTOR NEURONE EXCITABILITY IS REDUCED IN SOLEUS AND TIBIALIS ANTERIOR OF OLDER ADULTS
}

\author{
Running title: Intrinsic motor neurone excitability is reduced with ageing.
}

Lucas B. R. Orssatto ${ }^{1 *}$, 1.betdarosaorssatto@qut.edu.au, https://orcid.org/0000-0003-3788-3700;

David N. Borg 2 , david.borg@griffith.edu.au, https://orcid.org/0000-0002-0152-571X;

Anthony J. Blazevich ${ }^{3}$, a.blazevich@ecu.edu.au, https://orcid.org/0000-0003-1664-1614;

Raphael L. Sakugawa ${ }^{4}$, rlsakugawa@gmail.com, https://orcid.org/0000-0002-4162-232X;

Anthony J. Shield 1, aj.shield@qut.edu.au, https://orcid.org/0000-0002-0393-2466;

Gabriel S. Trajano 1, g.trajano@qut.edu.au, https://orcid.org/0000-0003-4667-4257.

${ }^{1}$ School of Exercise and Nutrition Sciences, Faculty of Health, Queensland University of Technology (QUT), Brisbane, Australia.

${ }^{2}$ Griffith University, Menzies Health Institute Queensland, The Hopkins Centre, Brisbane, Australia.

${ }^{3}$ Centre for Exercise and Sports Science Research (CESSR), School of Medical and Health Sciences, Edith Cowan University, Joondalup, Australia.

${ }^{4}$ Biomechanics Laboratory, Department of Physical Education, Federal University of Santa Catarina, Florianopolis, Brazil.

*Corresponding author: Lucas Bet da Rosa Orssatto; email: 1.betdarosaorssatto@qut.edu.au; https://orcid.org/0000-0003-3788-3700. School of Exercise and Nutrition Sciences, Faculty of Health, Queensland University of Technology (QUT), Brisbane, Australia. 


\section{ABSTRACT}

Age-related deterioration within both motor neurones and monoaminergic systems should theoretically reduce neuromodulation by weakening motor neuronal persistent inward current (PIC) amplitude. However, this assumption remains untested. Surface electromyographic signals were collected using two 32-channel electrode matrices placed on

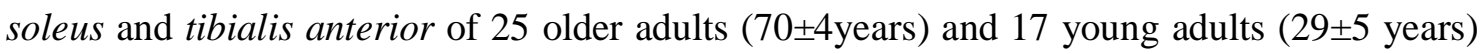
to investigate motor unit discharge behaviours. Participants performed triangular-shaped plantar and dorsiflexion contractions to $20 \%$ of maximum torque at a rise-decline rate of $2 \% / \mathrm{s}$ of each participant's maximal torque. Pairwise and composite paired-motor unit analyses were adopted to calculate delta frequency $(\Delta \mathrm{F})$, which has been used to differentiate between the effects of synaptic excitation and intrinsic motor neuronal properties and is assumed to be proportional to PIC amplitude. Soleus and tibialis anterior motor units in older adults had lower $\Delta F$ s calculated with either the pairwise $[-0.99$ and $-1.46 \mathrm{pps} ;-35.4$ and $33.5 \%$, respectively] or composite $(-1.18$ and $-2.28 \mathrm{pps} ;-32.1$ and $-45.2 \%$, respectively) methods. Their motor units also had lower peak discharge rates $(-2.14$ and -2.03 pps; -19.7 and $-13.9 \%$, respectively) and recruitment thresholds $(-1.50$ and $-2.06 \%$ of maximum, respectively) than young adults. These results demonstrate reduced intrinsic motor neurone excitability during low-force contractions in older adults, likely mediated by decreases in the amplitude of persistent inward currents. Our findings might be explained by deterioration in the motor neurones or monoaminergic systems and could contribute to the decline in motor function during ageing; these assumptions should be explicitly tested in future investigations.

Keywords: Persistent inward current; Ageing; Motor unit; HD-EMG; Motoneuron; Sarcopenia. 


\section{INTRODUCTION}

The age-related loss of force production has been comprehensively described in the literature [1-3], with the physiological alterations affecting force production including changes in several pathways within the nervous system $[1,4-6]$. The motor neurone is an important component of the nervous system affected by ageing as it is responsible for integrating and amplifying excitatory synaptic input into an appropriate motor output [7]. An essential intrinsic property of the motor neurone is its capacity to set up persistent inward currents (PICs), which are depolarising currents generated by voltage-sensitive sodium and calcium channels that increase cell excitability by amplifying and prolonging synaptic input $[8,9]$. Importantly, increases in the concentration of the monoamines serotonin and noradrenaline facilitate PIC development. Under conditions of high monoaminergic drive, synaptic input can be amplified by at least five-fold, suggesting that this amplification is a critical determinant of the motor neurone's ability to achieve the discharge rates observed during normal motor behaviour [10-12]. Thus, potential physiological alterations in motor neurone intrinsic properties, or in the monoaminergic input to the motor neurone, might reduce the motor neurone's ability to discharge at higher rates, thus reducing the ability to produce high muscle forces.

During ageing, several changes are observed in the motor neurones that might potentially reduce PIC amplitude in older adults, including lower discharge rates [13,14], reduced incidence of doublet discharges [15], and an increased afterhyperpolarisation duration [16]. These changes are consistent with the lower motor neurone excitability that is also observed in aged rat models [17,18]. With respect to the monoaminergic system, research using both human and animal models suggests that ageing is associated with reduced noradrenaline and serotonin secretions and thus input onto the motor neurones [19-23], which might theoretically underpin PIC amplitude reduction with ageing. These findings indicate the possibility that PIC amplitude might be reduced in older adults; however, this hypothesis remains to be tested [24].

The amplitude of PICs can be estimated in humans using the paired motor unit technique $[8,25,26]$, with data obtained using high-density surface electromyography $[27,28]$. This technique requires the pairing of the discharge rates of a low-threshold (control unit) to a higher-threshold (test unit) motor unit, obtained during a slowly-increasing and decreasing triangular-shaped contraction [8,25,29]. Subsequently, the difference in discharge rate of the control unit between the time of recruitment and de-recruitment of the test unit is computed as the change in frequency $(\Delta \mathrm{F}) . \Delta \mathrm{F}$ has been used to differentiate between the effects of 
synaptic excitation and motor neuronal intrinsic properties and is assumed to be proportional to PIC amplitude [9]. However, $\Delta \mathrm{F}$ values need to be interpreted with caution as they can be affected by spike frequency adaptation, spike frequency accommodation, and the proportion of sub-threshold PICs [8,30,31]. When controlling for these confounding factors, the technique can be used to estimate and compare PIC amplitudes in motor units of young and older adults.

The present study compared $\Delta \mathrm{F}$ amplitudes (i.e., estimates of PICs) of soleus and tibialis anterior motor units between young and older adults. Additionally, we explored the relationship between $\Delta \mathrm{F}$ and the peak discharge rates. We hypothesised that there would be a reduction in older adults' $\Delta \mathrm{F}$ in both soleus and tibialis anterior, and that $\Delta \mathrm{F}$ would be strongly associated with motor unit peak discharge rate. Soleus and tibialis anterior were selected for study because the control and timing of their activation are both critical to the performance of daily activities such as standing and walking in older adults $[32,33]$.

\section{METHODS}

\section{Participants and ethical procedures}

Forty-four participants were recruited for the study, including 18 young adults and 26 older adults (participant characteristics are documented in Table 1). More older adults were recruited because it was expected that fewer motor units would be identified during decomposition and that some participants may not be able to perform the triangle-shaped contractions with the necessary torque rise and fall accuracy. To participate, volunteers had to: a) be young adults aged 18 - 35 years or older adults $\geq 65$ years; b) have no history of neurological disorders; c) be free of lower limb musculoskeletal injuries; and d) not be taking medications that could influence the monoaminergic system, including serotonin or noradrenaline modulators (e.g., beta-blockers and serotonin reuptake inhibitors). Also, participants were instructed to not consume caffeinated foods (e.g., coffee) $24 \mathrm{~h}$ before the testing session. Participants were excluded from the analyses if: a) no usable motor units were identified by the decomposition algorithm, or b) if it was not possible to achieve all the assumptions required in the paired motor unit analysis (as described below), for at least one pair of motor units, for either soleus or tibialis anterior. One participant per group was excluded from the study because no motor units were identified in either soleus or tibialis anterior. The study was approved by the University Human Research Ethics Committee, and all participants gave written informed consent before participating. Data collection was 
117

118

119

120

121

conducted during the COVID-19 pandemic and all safety procedures followed the local state government policies.

Table 1. Participant characteristcs.

\begin{tabular}{|c|c|c|}
\hline Variable & Young Adults $(\mathrm{n}=17)$ & Older Adults $(\mathrm{n}=25)$ \\
\hline Age (years) & $29 \pm 5$ & $70 \pm 4$ \\
\hline \multicolumn{3}{|l|}{$\operatorname{Sex}(n, \%)$} \\
\hline Men & $9(53 \%)$ & $11(44 \%)$ \\
\hline Women & $8(47 \%)$ & $14(56 \%)$ \\
\hline Body mass (kg) & $71.9 \pm 13.6$ & $77.8 \pm 19.3$ \\
\hline Body fat (\%) & $20.2 \pm 7.7$ & $33.0 \pm 10.0$ \\
\hline Apendicular skeletal muscle mass $(\mathrm{kg})$ & $25.2 \pm 6.7$ & $21.6 \pm 5.1$ \\
\hline Height $(\mathrm{cm})$ & $175 \pm 11$ & $166 \pm 9$ \\
\hline Handgrip strength $(\mathrm{kg})$ & $40.3 \pm 13.3$ & $26.4 \pm 10.5$ \\
\hline \multicolumn{3}{|l|}{ Peak torque $(\mathrm{N} \cdot \mathrm{m})$} \\
\hline Plantar flexion & $156 \pm 47$ & $85 \pm 32$ \\
\hline Dorsiflexion & $41 \pm 14$ & $29 \pm 7$ \\
\hline \multicolumn{3}{|l|}{ Normalised peak torque $\left(\mathrm{N} \cdot \mathrm{m} \cdot \mathrm{kg}^{-1}\right)$} \\
\hline Plantar flexion & $2.13 \pm 0.49$ & $1.11 \pm 0.37$ \\
\hline Dorsiflexion & $0.56 \pm 0.09$ & $0.38 \pm 0.10$ \\
\hline \multicolumn{3}{|l|}{ Functional capacity } \\
\hline Timed up-and-go (s) & - & $6.1 \pm 1.0$ \\
\hline 5-times sit-to-stand (s) & - & $13.4 \pm 2.9$ \\
\hline Physical activity level (MET-min/week) & $2541(1902-3039)$ & $2994(1386-5139)$ \\
\hline
\end{tabular}

Note: Data are presented as mean \pm standard deviation, except for Physical activity level, which is presented as median with interquartile range. MET-min/week, metabolic equivalent of task score of the performed physical activities by the minutes accomplished per week.

\section{Study design and testing procedures}

Participants visited the laboratory on a single occasion in which they were familiarised with the testing procedures and data were collected. Initially, participants signed the informed consent form, and completed the International Physical Activity Questionnaire 
(short-version), which was used to estimate weekly physical activity levels based on the metabolic equivalent of a task (MET - i.e., multiples of the resting metabolic rate) for each physical activity domain. MET scores for each activity were multiplied by the minutes performed in one week, providing the total MET-min/week [34,35]. Physical activity levels were interpreted based on the recommendations of the Guidelines for Data Processing and Analysis of the International Physical Activity Questionnaire (IPAQ) [35]. Thereafter, body composition assessment was conducted with a multi-frequency bioelectrical impedance device (MC-780, Tanita, Japan), which provided body fat percentage and appendicular skeletal muscle mass data.

After electrode placement on soleus and tibialis anterior, the participants were seated upright in the chair of an isokinetic dynamometer (Biodex System 4, Biodex Medical system, Shirley, NY) with the knee fully extended $\left(0^{\circ}\right)$ and ankle in the anatomical position $\left(0^{\circ}\right)$. A warm-up consisting of six 5-s submaximal voluntary isometric plantar and dorsiflexion contractions $(2 \times 30 \%, 2 \times 60 \%$, and $2 \times 80 \%$ of perceived maximal effort) was performed, followed by three maximal voluntary contractions of $\sim 3$-s with 30 -s rest intervals. The maximum torque achieved was recorded as the maximal voluntary contraction peak torque, which was also normalised to body mass. Subsequently, participants were familiarised with the triangular-shaped contractions to $20 \%$ of their maximum voluntary torque level. Triangular contractions to $20 \%$ of maximal torque have been extensively used for $\Delta \mathrm{F}$ calculations using the paired motor unit technique [8,31,36-38], and this force was considered similar to the average torques developed during daily activities such as standing [39] and walking. All contractions had a duration of $20 \mathrm{~s}$ (10-s up and 10-s down) and were performed at a rate of torque increase and decrease of $\sim 2 \% / \mathrm{s}$. Participants were instructed to follow the torque path provided in real time on a $58-\mathrm{cm}$ computer monitor during each contraction. Data collection commenced $5 \mathrm{~min}$ after the end of familiarisation (usually requiring $\sim 3-10 \times 20 \%$ triangular contractions with 30-s rest), during which the participants then performed four triangular contractions with 60-s rest intervals. When an abrupt increase or decrease in torque was observed (i.e., the torque trajectory was not closely followed), the trial was excluded and repeated. The maximum voluntary isometric torque and order of triangular contraction completion was randomised between soleus and tibialis anterior.

After the neuromuscular assessments, the participants performed a handgrip strength test using a grip force transducer (ADinstruments, Australia). They performed 3 submaximal (50\% of their perceived maximal effort) familiarisation contractions, followed by $3 \times 3-\mathrm{s}$ maximal contractions with 30-s rest intervals. The maximum force achieved was recorded as 
their handgrip strength. Thereafter, they performed sit-to-stand and timed up-and-go (functional capacity) tests, timed off-line with video recordings as recommended by da Silva et al. [40] to reduce measurement error. The 5-times sit-to-stand required the participants to stand up five times until they reached upright standing and then returned to the seated posture on a chair (seat $46 \mathrm{~cm}$ high). The timed up-and-go test required the participants to rise from a chair (seat $46 \mathrm{~cm}$ high), walk towards and around a cone $3 \mathrm{~m}$ from the chair, return to the starting position, and to sit without the aid of hands and not running, in the shortest possible time. The fastest of three attempts (60-s rest between) was analysed.

The participants' sarcopaenia status was screened according to the cut-off points and algorithm from the European Working Group on Sarcopenia in Older People (EWGSOP2) [48]. According to this scale, older adults with low handgrip strength ( $<27 \mathrm{~kg}$ for men or $<16$ $\mathrm{kg}$ for women) are classified as "sarcopaenia probable", and if this is accompanied by low appendicular skeletal muscle mass ( $<20 \mathrm{~kg}$ for men and $<15 \mathrm{~kg}$ for women) then they are confirmed as sarcopaenic. Older adults with confirmed sarcopenia who take longer than $20 \mathrm{~s}$ to perform the timed up-and-go test are classified as having severe sarcopaenia. Also, participants with timed up-and-go performance under $12 \mathrm{~s}$ are classified as having normal mobility [41].

\section{Surface electromyography}

Surface electromyograms (sEMG) were recorded during the $20 \%$ triangular contractions using four semi-disposable 32-channel electrode grids with a 10-mm interelectrode distance (ELSCH032NM6, OTBioelettronica, Torino, Italy). After skin shaving, abrasion, and cleansing with 70\% isopropyl alcohol, two electrode grids were placed over the medial and lateral portions of soleus (either side of the Achilles tendon) and another two electrode grids were placed over the superior and inferior aspect of tibialis anterior using a bi-adhesive foam layer and conductive paste (Ten20, Weaver and Company, Colorado, USA). A strap electrode (WS2, OTBioelettronica, Torino, Italy) was dampened and positioned around the ankle joint as a ground electrode. The sEMG signals were acquired in monopolar mode, amplified (256×), band-pass filtered $(10-500 \mathrm{~Hz})$, and converted to a digital signal at $2048 \mathrm{~Hz}$ by a 16-bit wireless amplifier (Sessantaquattro, OTBioelettronica, Torino, Italy) using OTBioLab+ software (version 1.3.0., OTBioelettronica, Torino, Italy) before being stored for offline analysis.

\section{Motor unit analyses}




\section{Motor unit identification}

The recorded data were processed offline using the DEMUSE software [27]. For each muscle, only the triangular contraction yielding the lowest deviation from the torque trajectory was analysed. If both contractions presented a similar torque trajectory, the contraction with the highest number of identified motor units was analysed. High-density sEMG signals were band-pass filtered $(20-500 \mathrm{~Hz})$ with a second-order, zero-lag Butterworth filter. Thereafter, a blind source separation method, the convolutive kernel compensation (CKC) method, was used for signal decomposition [27,42] from each triangular contraction. CKC yields the filters of individual motor units (so-called motor unit filters) that, when applied to high-density sEMG signals, estimate the motor unit spike trains [27,42]. After decomposition, a trained investigator manually inspected motor unit spike trains and edited the discharge patterns of the motor units. Only the motor units with a pulse-to-noise ratio equal to or greater than $30 \mathrm{~dB}$ were kept for further analysis [42].

\section{Estimation of PIC amplitude $(\Delta F)$ and peak discharge rate}

The observed discharge events for each motor unit were converted into instantaneous discharge rates and fitted into a $5^{\text {th }}$-order polynomial function. The maximum value obtained from the polynomial curve was considered the peak discharge rate. The relative torque $(\%)$ produced at the time in each motor unit was recruited was considered the recruitment threshold. The recruitment threshold was used to characterise the populations of motor units identified by the decomposition algorithm for each group. Thereafter, PIC amplitude was estimated using the paired motor unit analysis [8], referred through the manuscript as pairwise method. Motor units with a low recruitment threshold (i.e., control units) were paired with higher recruitment threshold motor units (i.e., test units). $\Delta \mathrm{F}$ was calculated as the change in discharge rates of the control motor unit from the moment of recruitment to the moment of de-recruitment of the test unit [8,9]. In order to produce motor unit pairs, the following criteria were adopted: 1) rate-to-rate correlations between the smoothed discharge rate polynomials of the test and control units was $r \geq 0.7 ; 2$ ) test units were recruited at least $1.0 \mathrm{~s}$ after the control units; and 3) the control unit did not show discharge rate saturation after the moment of test unit recruitment (i.e., discharge rate from the control unit at the moment the test unit was recruited minus the peak discharge rate at the control unit $>0.5 \mathrm{pps}$ ) $[8,38,43-45]$. $\Delta$ Fs obtained for each control unit were averaged to obtain a single $\Delta \mathrm{F}$ for each test motor unit. 
231 We also conducted an additional analysis using the composite paired motor unit 232 method to calculate $\Delta \mathrm{F}$ values for each motor unit [31]. This method is characterised by the 233 overlay of 3 lower-threshold motor units to construct a single composite control unit profile 234 to be paired with the test units. The composite method has been suggested to address some of 235 the limitations observed with the pairwise method, such as underestimation and 236 overestimation of $\Delta \mathrm{F}$ values, reducing its variability. However, strict assumptions are made 237 for eligible motor units to be included in the analysis, which allows its use only in muscles in 238 which it is possible to identify many motor units (e.g., tibialis anterior) but not in those in 239 which fewer motor units are identified using the decomposition method (e.g., soleus). 240 Moreover, this method does not allow calculation of $\Delta \mathrm{F}$ values for lower-threshold motor 241 units since they are used to construct the composite control unit. In summary, the control unit 242 is the overlay of the instantaneous discharge rate of at least three motor units recruited at $<3 \%$ 243 of the maximum voluntary torque and presenting a similar discharge profile, which was 244 determined visually by an experienced researcher with individual motor units discharge rates 245 plotted superimposed to each other. This is followed by the application of the $5^{\text {th }}$ order 246 polynomial over the overlayed motor units discharge rates. It was assumed that lower247 threshold motor units had their discharge rate profile more linearly related to the synaptic 248 input profile because PICs were almost fully activated at the time of recruitment; therefore, it 249 would be more appropriate to use the composite unit as control. Also, this method requires 250 the removal of the acceleration phase of the discharge rates (i.e., secondary range), making 251 the polynomial ascending-to-descending slope ratio near 1, which is important when 252 measuring PIC amplitudes to avoid any rate-dependent effects on motor unit recruitment or 253 derecruitment [46]. The secondary range was determined with visual inspection of deflection 254 point following Afsharipour et al., [31] procedures. The test units should start discharging in 255 the tertiary range (i.e., after the secondary range and before the descending phase). This 256 method provides a single $\Delta \mathrm{F}$ for each motor unit, which was then used in the data analysis. 257 Figure 1 illustrates the pairwise and composited paired motor unit analysis methods on 258 tibialis anterior motor units for one participant per group. Panels C and D display the test 259 units used for both methods. Panels E and F display the control units for the pairwise method 260 and panels $\mathrm{G}$ and $\mathrm{H}$ display the control units for the composite method. 

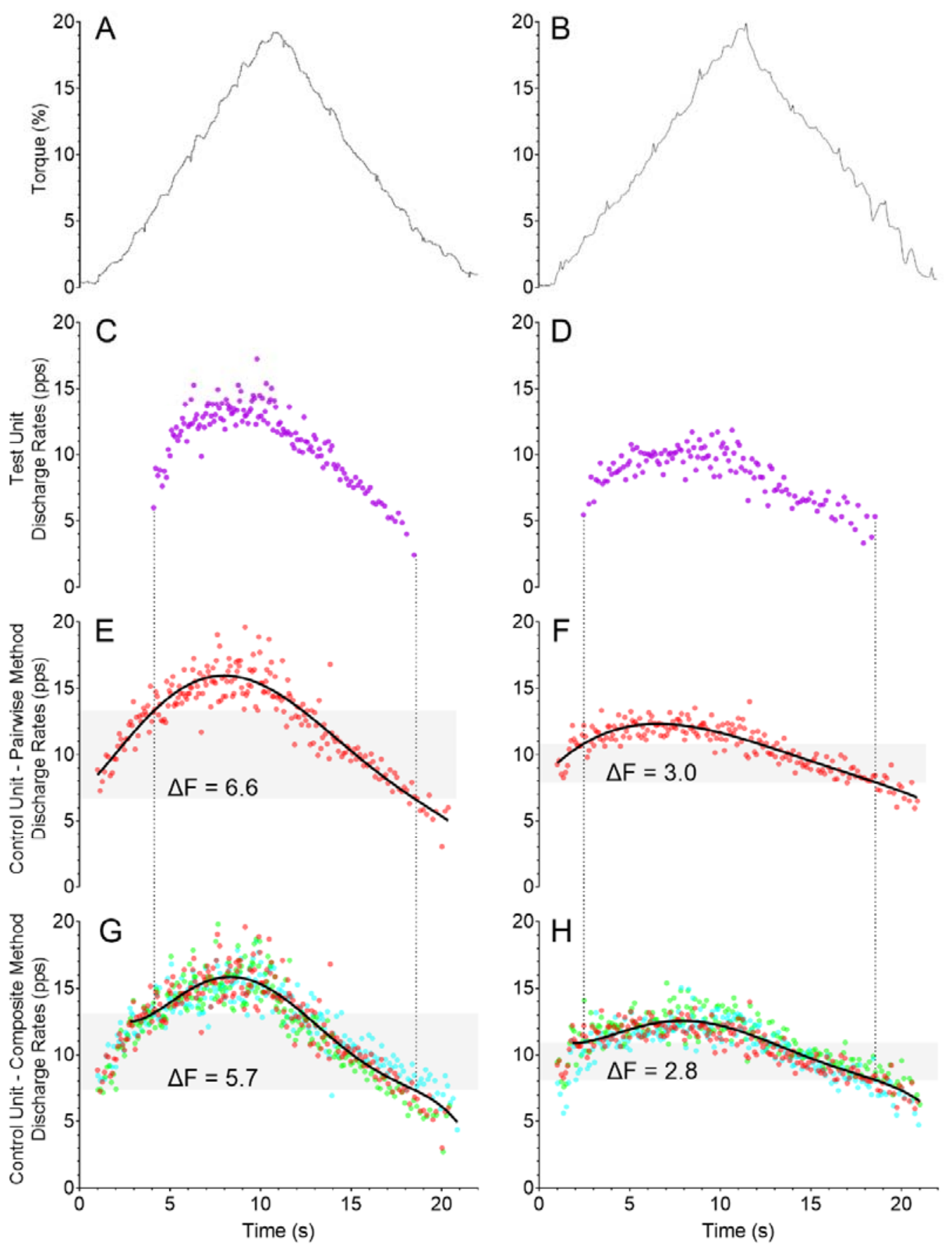

263 Figure 1. Data from a single participant for each group showing torque during triangleshaped contractions and delta frequency $(\Delta \mathrm{F})$ calculation in tibialis anterior for both the pairwise and composite paired motor unit analyses. Data from a young adult is displayed in the left panels and from an older adult in the right panels. Panels A and B on the first row show the torque traces for contractions with $20 \%$ of the participant's maximal voluntary torque. The participants' test units are displayed on panels C and D (purple motor unit), 
269

270

271

272

273

274

275

control units for the pairwise method on panels $\mathrm{E}$ and $\mathrm{F}$ (red motor unit), and control units for the composite method on panels $\mathrm{G}$ and $\mathrm{H}$ (red, green, and blue motor units). The black continuous lines are the $5^{\text {th }}$-order polynomial fits for the control units. Note that for the composite method the polynomial curve starts from the tertiary range. The gray-shaded areas represent the $\Delta \mathrm{F}$ amplitude for each participant and analysis.

\section{Data analysis}

All analyses were undertaken in $\mathrm{R}$ (version 4.0.3) using RStudio environment (version 1.3.1093). Models were fitted using the lmerTest package [47]. A linear mixedeffects model was used to compare estimates of $\Delta \mathrm{F}$ of soleus and tibialis anterior motor units between young and older adults. The model included: age group, muscle type and recruitment threshold as fixed effects. A random intercept and correlated random slopes (recruitment threshold and muscle type) were included for each participant in the study, to account for the correlation between repeated observations on each individual. This model was selected from a series of candidate models (Supplement 1), based on the smallest Bayesian Information Criteria value. The recruitment threshold was standardised (mean $=0, \mathrm{SD}=1$ ) before analysis.

Separate linear mixed-effects models were used to analyse peak discharge rate and recruitment threshold data. These models included: age group, muscle type and age group by muscle type as fixed factors; and a random intercept and slope (muscle group) for each participant. The estimated marginal mean difference and $90 \%$ and $95 \%$ confidence intervals (CI) in $\Delta \mathrm{F}$, peak discharge rate, and recruitment threshold between young and older adults, were determined using the emmeans package [48]. The standardised difference, denoted $d$, was also calculated using the population SD from each respective linear mixed-effects model as the denominator [48].

To determine the contribution of $\Delta \mathrm{F}$ to peak discharge rate, a linear mixed-effects model was fitted, with the coefficient of determination $\left(\mathrm{R}^{2}\right)$ used to quantify the proportion of the variance in peak discharge rate explained by $\Delta \mathrm{F}$ [49]. The model included $\Delta \mathrm{F}$ as a fixed effect and a random intercept and slope $(\Delta \mathrm{F})$ for each participant, to account for the correlation of repeated measurements on an individual. $\Delta \mathrm{F}$ was standardised (mean $=0, \mathrm{SD}=$ 1). Differences between young and older adults in peak plantar flexion and dorsiflexion torque, and physical activity levels, were determined using independent $t$-tests. The $\alpha$ level 
301

302

303

304

for all tests was $5 \%$. The dataset and R code can be found at https://github.com/orssatto/PICsageing.

\section{RESULTS}

\section{Effects of age and muscle group on $\Delta F$, peak discharge rate, and recruitment threshold}

Motor units of older adults identified by the decomposition algorithm in our study had lower $\Delta$ Fs and peak discharge rates and were recruited at lower torque (muscle force) levels than young adults in both soleus and tibialis anterior (Figure 2, panels A, B, and C, respectively). Also, $\Delta \mathrm{F}$ levels and peak discharge rates were lower in soleus than tibialis anterior, independent of age (Figure 2, panels A and B).

There were effects of age $(\beta=-0.99,95 \% \mathrm{CI}=-1.42,-0.57 ; p<.001)$, muscle $(\beta=$ $1.56,95 \% \mathrm{CI}=1.05,2.07 ; p<.001)$ and recruitment threshold $(\beta=0.47,95 \% \mathrm{CI}=0.27$, $0.66 ; p<.001)$ but no age group by muscle effect $(\beta=-0.47,95 \% \mathrm{CI}=-1.15,0.21 ; p=.18)$, on $\Delta \mathrm{F}$ when calculated using the pairwise paired motor unit method. $\Delta \mathrm{F}$ was lower in older adults (Figure 2A) in both soleus $(d=-0.93$; Figure $3 \mathrm{~A})$ and tibialis anterior $(d=-1.38$; Figure 3A). There were effects of age $(\beta=-2.14,95 \% \mathrm{CI}=-2.98,-1.33 ; p<.001)$ and muscle $(\beta=3.71,95 \% \mathrm{CI}=2.64,4.83 ; p<.001)$ on peak discharge rate, but there was no age by muscle effect on peak discharge rate $(\beta=0.11,95 \% \mathrm{CI}=-1.37,1.56 ; p=.88)$. Peak discharge rate was lower in older adults (Figure 2B) in both soleus $(d=-0.98$; Figure $3 \mathrm{~B})$ and tibialis anterior $(d=-1.27$; Figure 3B). There was an age effect on recruitment threshold, with thresholds lower in older adults $(\beta=-1.50,95 \% \mathrm{CI}=-2.89,-0.12 ; p=.040$; Figure $3 \mathrm{C})$. There was no evidence of muscle $(\beta=1.12,95 \% \mathrm{CI}=-0.35,2.65 ; p=.15)$ or age by muscle $(\beta=1.12,95 \% \mathrm{CI}=-2.59,1.38 ; p=.58)$ effects on recruitment threshold.

The results of the additional, composite method, analysis revealed there was effects of age $(\beta=-1.18,95 \% \mathrm{CI}=-1.85,-0.51 ; p=.002)$, muscle $(\beta=1.37,95 \% \mathrm{CI}=0.74,2.00 ; p<$ $.001)$, and age by muscle $(\beta=-1.10,95 \% \mathrm{CI}=-1.39,-0.27 ; p=.014)$ on $\Delta \mathrm{F}$. $\Delta \mathrm{Fs}$ were lower in older adults in both soleus $(d=-1.54)$ and tibialis anterior $(d=-2.98)$, as shown in Figure 4. Also, $\Delta \mathrm{Fs}$ were lower in soleus than tibialis anterior in young adults (mean difference $=-1.37,95 \% \mathrm{CI}=-2.27,-0.46 ; p=.008 ; d=-1.79$ ) but not older adults (mean difference $=-0.27,95 \% \mathrm{CI}=-1.04,0.51 ; p=.47 ; d=-0.35)$. The composite method removed the effect of recruitment threshold on $\Delta \mathrm{F}(\beta=-0.05, p=.61)$ that was observed when modelling the pairwise method data $(\beta=0.47, p<.001)$. 

when accounting for the correlation between repeated observations on each participant, $\Delta \mathrm{F}$ explained $53 \%$ of the variance in peak discharge rate. There was a statistical effect of $\Delta \mathrm{F}$ on peak discharge rate $(\beta=1.19,95 \% \mathrm{CI}=0.87,1.51, p=.001)$.

(A)

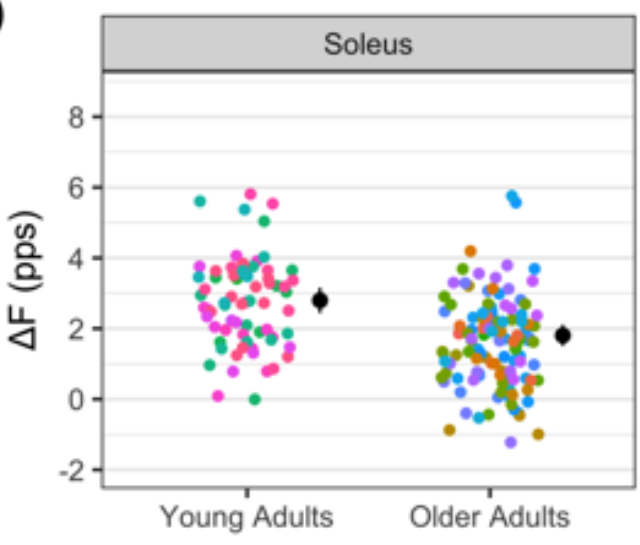

(B)

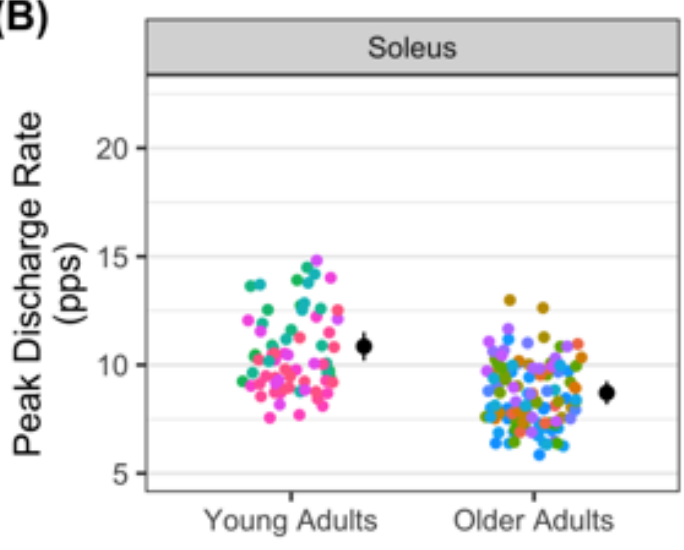

(C)

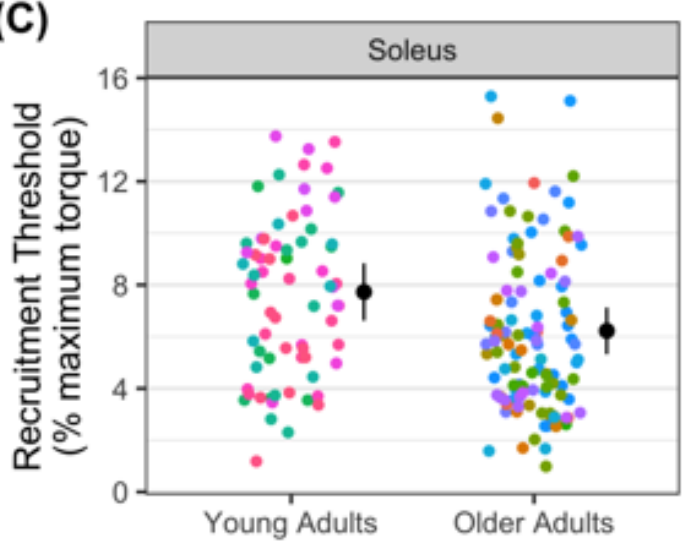

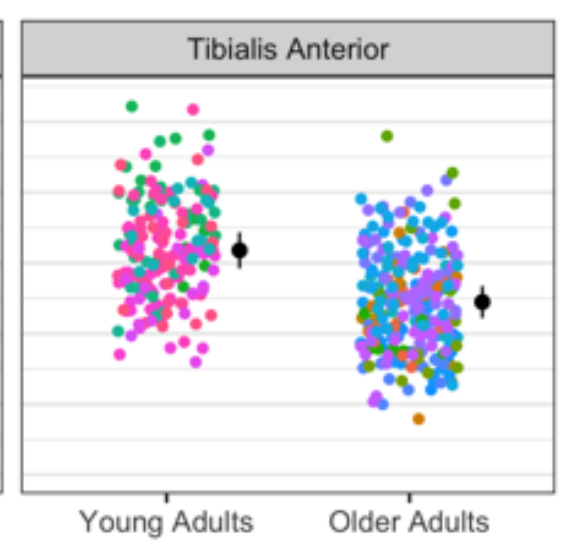
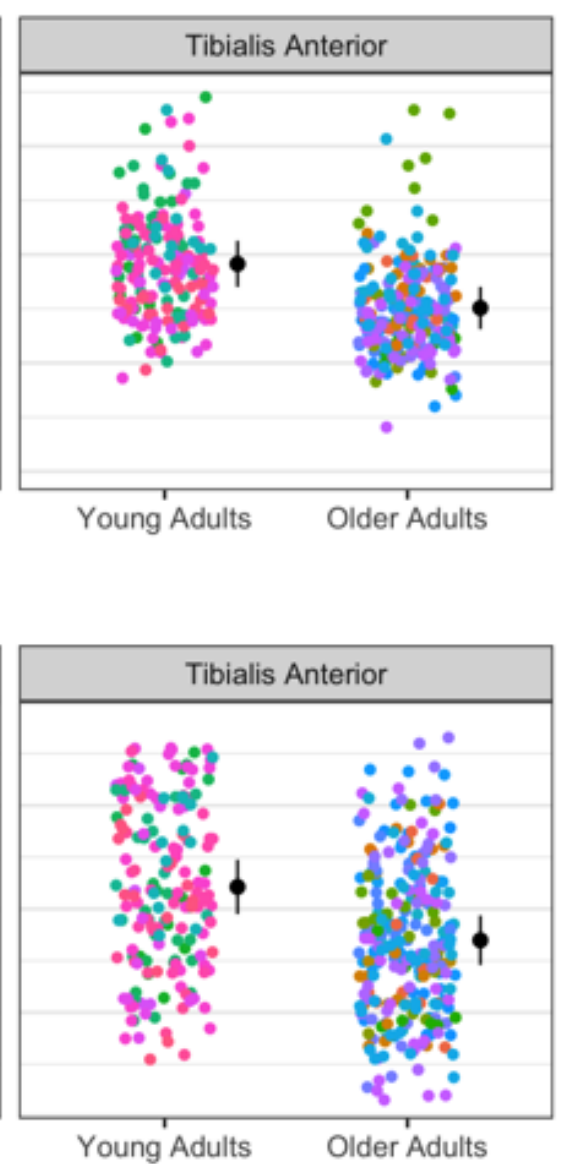

338 Figure 2. $\Delta \mathrm{F}$ calculated with the pairwise paired motor unit method (A), peak discharge rate

339 (B), and recruitment threshold (C) in soleus and tibialis anterior in young and older adults. 
340 The mean (circle) and 95\% confidence interval are offset to the right, with individual data

341 points coloured by participant. pps = peaks per second.

342

343

344
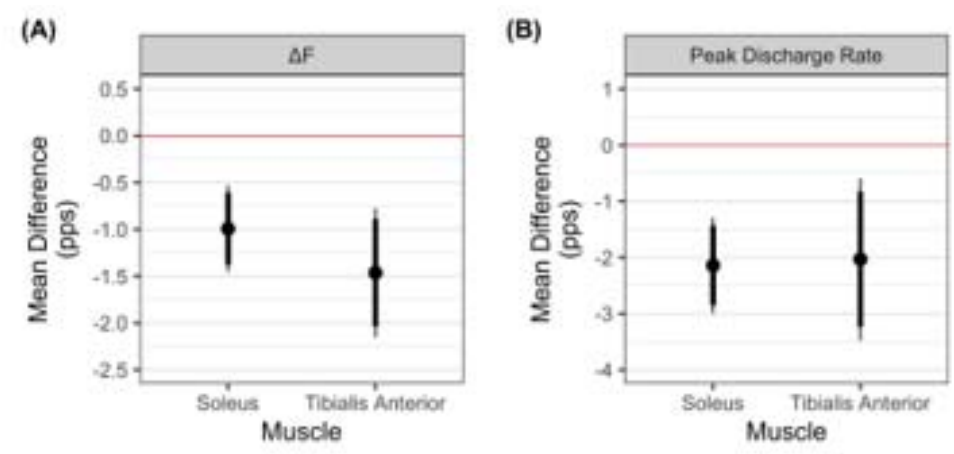

Figure 3. The marginal mean difference with $90 \%$ (thick line) and $95 \%$ (thick line) confidence intervals between young and older adults for $\Delta \mathrm{F}$ calculated with the pairwise paired motor unit method (A), peak discharge rate (B), and recruitment threshold (RT) (C) in soleus and tibialis anterior. Negative values on all panels indicate the lower responses in older adults. There was an effect of age on $\Delta \mathrm{F}$, peak discharge rate, and recruitment threshold, with all variables lower in older adults. There was no age by muscle effect on $\Delta \mathrm{F}$, peak discharge rate, or recruitment threshold-indicating that age-dependent differences in these variables were similar for both muscles.

(A)

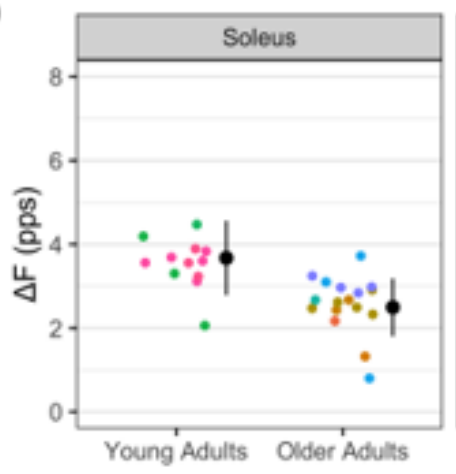

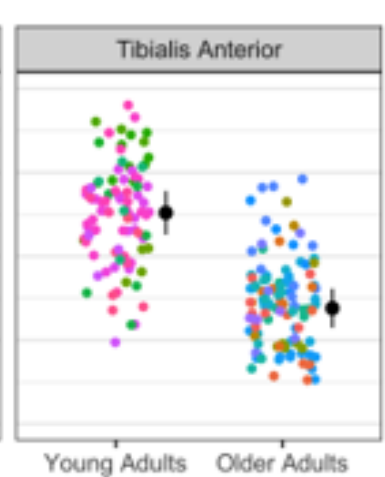

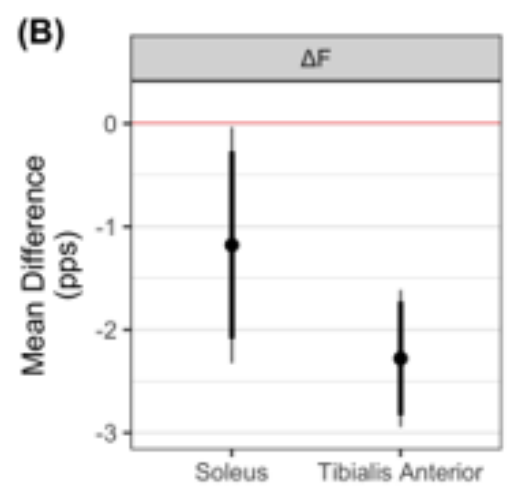

Figure 4. $\Delta \mathrm{F}$ calculated with the composite paired motor unit method (A), and the marginal mean difference with $90 \%$ (thick line) and 95\% (thick line) confidence intervals between young and older adults for $\Delta \mathrm{F}(\mathrm{B})$. In panel $\mathrm{A}$, the mean (circle) and $95 \%$ confidence interval are offset to the right, with individual data points coloured by participant. In panel B, negative values indicate $\Delta \mathrm{F}$ was lower responses in older adults. $\mathrm{pps}=$ peaks per second. 


\section{Participant characteristics}

Participant characteristics are shown in Table 1. Body fat (\%) was higher (mean difference $=12.7 \%$ of body mass, $95 \% \mathrm{CI}=7.1,18.4 ; \mathrm{p}<.001 ; d=1.40)$ and appendicular skeletal muscle mass was lower (mean difference $=-3.7 \mathrm{~kg}, 95 \% \mathrm{CI}=-7.3,-0.1 ; p=.045 ; d$ $=-0.63$ ) in older adults than young adults. Handgrip strength was lower in older adults (mean difference $=-14.0 \mathrm{~kg}, 95 \% \mathrm{CI}=-21.2,-6.7 ; p<.001 ; d=-1.19)$. Absolute peak torque was lower in older adults in both plantar flexion (mean difference $=-70 \mathrm{~N} \cdot \mathrm{m}, 95 \% \mathrm{CI}=-95,-46$; $p<.001 ; d=-1.83$ ) and dorsiflexion (mean difference $=-12 \mathrm{~N} \cdot \mathrm{m}, 95 \% \mathrm{CI}=-18,-5 ; p<$ $.001 ; d=-1.18$ ), as was the normalised peak torque in both plantar flexion (mean difference $=-1.02 \mathrm{~N} \cdot \mathrm{m} \cdot \mathrm{kg}^{-1}, 95 \% \mathrm{CI}=-1.28,-0.77 ; p<.001 ; d=-2.45$ ) and dorsiflexion (mean difference $\left.=-0.17 \mathrm{~N} \cdot \mathrm{m} \cdot \mathrm{kg}^{-1}, 95 \% \mathrm{CI}=-0.23,-11 ; p<.001 ; d=-1.81\right)$.

According to the EWGSOP2 sarcopaenia cut-off points and algorithm [50], no participant was confirmed as sarcopaenic. Three older men and 1 older woman were classified as "sarcopaenia probable" (handgrip strength $<27 \mathrm{~kg}$ for men and $<16 \mathrm{~kg}$ for women), but their appendicular skeletal muscle mass levels were higher than the cut-off points, not confirming sarcopaenia (>20 kg for men or $>15 \mathrm{~kg}$ for women). All older adults performed the timed up-and-go test in $<12 \mathrm{~s}$ and were classified as normal mobility.

Physical activity levels were not statistically different between young and older adults (mean difference $=594 \mathrm{MET}$ min/week, 95\% CI $=-1269,2457 ; p=.52 ; d=0.20$ ), with both young and older adults presenting with moderate-to-high physical activity levels.

\section{Motor unit identification}

The number of motor units identified in soleus was 113 for the young group and 211 for the older group. The median (IQR) number per younger participant was 7 (5-8) and per older participant 8 (6-9). For tibialis anterior, 293 motor units were identified in the young group and 411 in the older group. The median number per younger participant was 19 (16-23) and per older participant 20 (16-27). For the pairwise paired motor unit analysis, it was possible to obtain $\Delta \mathrm{F}$ values from 16 young adults and 23 older adults in soleus, and from 16 young adults and 19 older adults in tibialis anterior. The number of test unit $\Delta F$ s for soleus in the young group was 70 and in the older group was 117. The median number per young participant was 5 (3-6) and per older participant was 4 (3-6). The number of test unit $\Delta \mathrm{Fs}$ for tibialis anterior in the young group was 185 and in the older group was 257 . The median number per younger participant was $11(8-14)$ and per older participant was 14 (9$18)$. 
394

395

For the composite paired motor unit analysis, it was possible to obtain $\Delta \mathrm{F}$ values from 4 young adults and 7 older adults for soleus and from 15 young adults and 18 older adults for tibialis anterior. The number of test unit $\Delta \mathrm{Fs}$ for soleus in the young group was 12 and in the older group was 17, with the median number per younger participant being 3 (3-4) and per older participant being $2(1-4)$. The number of test unit $\Delta \mathrm{Fs}$ for tibialis anterior in the young group was 93 and in the older group was 111, with the median number per young participant being 5 (4-8) and per older participant being 6 (3-8). No motor units were identified in one young participant and one older participant in soleus, and in one young participant and six older participants in tibialis anterior, respectively. These participants were not included in the analyses for the respective muscles.

\section{DISCUSSION}

The present study compared amplitudes of motor neuronal PICs, estimated using the paired motor unit technique $(\Delta \mathrm{F})$, between young and older adults. The main findings indicate that $\Delta \mathrm{F}$ values are considerably lower in both soleus and tibialis anterior in older adults. These reductions are accompanied by lower peak discharge rates and the recruitment of motor units at lower torque levels in the older than younger adults in both muscles. As an exploratory analysis, a model was developed that showed a small contribution of $\Delta \mathrm{F}$ to the between-subject variance in peak discharge rates. These findings point to a meaningful reduction in the intrinsic motor neurone excitability in a group of non-sarcopaenic older adults with normal mobility that is at least partly associated with a reduced amplitude of persistent inward currents.

\section{Estimates of persistent inward currents $(\Delta F)$}

The $\Delta \mathrm{F}$ values obtained from soleus and tibialis anterior in young adults are similar to those obtained in previous studies using triangular-shaped contractions with a $2 \% / \mathrm{s}$ force increase-decrease rate $[31,36,37]$ and indicate that $\Delta F$ from older adults are substantially lower than in young adults in both soleus and tibialis anterior. However, our results partially differ from previous findings that investigated self-sustained discharging in older humans [51] and the frequency-current slope in aged rats [18]. Kamen et al., [51] observed no difference between young and older adults in self-sustained discharging of tibialis anterior motor units when excitatory input was applied by delivering a brief vibration stimulus. In addition, they have observed no differences between age groups in maximal voluntary force and no change in force caused by the vibration stimulus. These results could indicate that the 
428

429

430

431

432

433

434

reductions in PICs with ageing seem clearer when tested with the paired-motor-unit analysis, or because older adults present significantly lower force levels than their younger counterparts. In agreement to our result, Kalmar et al., [18] observed lower frequency-current slope, which indicate lower PICs amplitude, in older rats hindlimb muscles. However, they have also reported higher incidence of PICs in older rats, which could be an adaptative response to counteract the reductions in PICs amplitude, justified by augmentation of the serotonin and noradrenaline receptor sensitive to residual endogenous monoamines [52].

Weaker PICs in older adults can be a result of the detrimental changes within the monoaminergic systems, which indicate lower release of serotonin and noradrenaline with ageing. Locus coeruleus and the dorsal raphe nucleus are the major central sources of noradrenaline and serotonin, respectively [53]. Diminution of structural integrity [19,54] and in neuromelanin (a product of noradrenaline synthesis) content in noradrenergic neurones emanating from locus coeruleus [20] indicate impaired noradrenaline secretion in older adults. Moreover, noradrenaline and serotonin concentration decrements have been observed in the brains of aged rats [21,22], and degeneration of serotonergic axons projecting to the ventral horn of the lumbar segment of the spinal cord (where motor neurones innervating lower limb muscles emanate) have been detected [23]. Also, serotonin receptors are affected by expanded circulation of cytokines, resulting in increased re-uptake of serotonin [55]. Thus, older adults might speculatively present reduced noradrenaline and serotonin secretions, and hence input onto motor neurones, which would then impair the initiation and modulation of PICs in this population. Changes in motor neurone integrity might also partly underpin reductions in intrinsic excitability. During ageing, axonal demyelination due to reduced expression of proteins responsible for myelination [56] as well as axonal atrophy and degeneration have been observed, possibly subsequent to deregulated $\mathrm{Ca}^{2+}$ homeostasis [57] and to toxic, metabolic, or infectious injury sustained throughout the lifespan, or due to high levels of chronic inflammation and oxidative stress [58,59]. Motor neuronal death, especially in higher-threshold motor axons, leading to denervation of motor units has also been documented [60]. In these cases, denervated motor units may remodel through reinnervation by nearby lower-threshold motor neurones [4,61], which may explain the reduced recruitment threshold observed in the older adults (as discussed below). These detrimental alterations in motor neurone structure are associated with reduced $\mathrm{Ca}^{2+}$-mediated plateau potential durations in striatal neurones (from aged rats) [62], slower conduction velocity of efferent motor axons [63], lower incidence of doublet discharges, slower maximum discharge rates $[13,14,64]$ alongside an increased afterhyperpolarisation duration [16], and, as evidenced in 
the present study, lower PIC amplitude in the motor neurones. It is a logical hypothesis that the changes within the monoaminergic system and motor neurone structural integrity would possibly explain the reduced $\Delta \mathrm{Fs}$ observed in the present study. However, our findings indicate the need for further study of the cause-effect relationship between these mechanisms and reduced PICs in humans.

PICs are highly sensitive to synaptic inhibition, and both reciprocal and recurrent inhibition directly influence intrinsic motor neurone excitability, being effective PIC deactivators by opposing the facilitatory effects on PICs of descending brainstem neuromodulatory systems [65-69]. It would therefore be expected that the reduced PIC amplitude estimates observed in the older adults in the present study might result from an age-dependent increase in reciprocal and/or recurrent inhibition. However, older adults present reduced reciprocal inhibition from the common peroneal nerve onto soleus and from the tibial nerve onto the tibialis anterior [70]. Also, recurrent inhibition onto the soleus motor neurone pool is suspected to be relatively unaffected by ageing [71]. Therefore, a reasonable supposition is that the behaviour of spinal inhibitory/excitatory systems (i.e., reduced reciprocal inhibition and preserved recurrent inhibition) of older adults are unlikely to be responsible for the decreases in PIC amplitude.

The age-related reductions in PIC amplitudes indicated by our data may partly explain losses in motor function with ageing, and may thus have important clinical practice implications. Motor neurone PICs can amplify the synaptic input they receive, allowing motor neurones to discharge at higher rates, as shown in animal, computational modelling, and human studies [10,11,29,72]. This amplification system is an important feature when higher-intensity muscular contractions are needed. Thus, weaker motor neurone PICs could be an important limiting factor which to some extent explains the lower voluntary activation levels [73] and force production [2] observed in older adults. A lower capacity for producing high forces as a result of weaker PICs could therefore account for the increase in relative intensity and level of effort needed for older adults when performing daily activities [74]. This would indicate a reduced performance and increased fatigability when performing these activities [75]. Moreover, if these assumptions are true then the improvements in force and functional capacity following resistance training in older adults [76,77] could be partly mediated by increases in PIC amplitudes [24]. However, these causal hypotheses remain untested.

\section{Recruitment threshold}


Motor units that were identified in the older adults in our study showed a lower recruitment threshold and were thus recruited at a lower torque level than in young adults.

498 This may be a result of two different factors: 1) the decomposition algorithm may have biased the identification of motor units towards the lower-threshold ones. Older adults have a greater proportion of lower-threshold motor units as a result of motor unit remodelling subsequent to motor neuronal denervation, so motor units previously innervated by higherthreshold motor units become reinnervated by lower-threshold motor units [61]. Therefore, the decomposition may have picked up more low-threshold motor units in older adults, whereas the higher-threshold units in young may bias the decomposition the opposite direction. 2) The observed lower recruitment thresholds might reflect the recruitment of a greater relative number of smaller motor neurones during the task. Since motor unit discharge rate modulation in response to force changes is impaired in older adults, additional motor units must be recruited earlier in a triangular-shaped contraction to continually increase muscle force $[78,79]$. If this is the case, our data in slow isometric contractions indicate that aged motor neurones have a constrained capacity to amplify the excitatory synaptic input, consequently demanding an earlier recruitment of additional motor units to achieve the required motor output.

It is important to note that $\mathrm{Ca}^{2+}$ PIC channels can be activated below the action potential threshold (i.e., subthreshold PICs), strongly influencing motor unit recruitment [80]. The possibility exists that the population of lower recruitment threshold motor units identified by the decomposition algorithm in the older adults presents a higher motor neurone excitability (i.e. enhanced motor neurone recruitment for a given input) because of stronger subthreshold PIC activation. An increase in the proportion of subthreshold PICs would reduce the recruitment threshold while also decreasing $\Delta \mathrm{F}$ values. Subthreshold PICs may be stronger in smaller motor neurones, resulting in a larger overall (sub- plus supra-threshold) PIC amplitude [31]. However, the $\Delta \mathrm{F}$ method only estimates the suprathreshold contribution of the PICs to the discharge behaviour of motor units. Consequently, it is not possible to ascertain the behaviour of subthreshold PICs using the $\Delta \mathrm{F}$ method. Our analytical approach accounted for any effect of recruitment threshold (and possibly motor neurone size) on $\Delta \mathrm{F}$, that may otherwise confound any motor-unit-population-related effect, by including recruitment threshold in our modelling of $\Delta \mathrm{F}$. Further, the reanalysis of $\Delta \mathrm{F}$ using the composite paired motor unit method appeared to remove the effect of recruitment threshold on $\Delta \mathrm{F}$ values (from $\beta=0.47, \mathrm{p}<.001$ to $\beta=-0.05, \mathrm{p}=.61$ ), which is an expected 
characteristic of the method [31]. Therefore, the reduced $\Delta \mathrm{Fs}$ observed in older adults in the present study is not likely to have been an artefact of the different populations of motor units identified between groups.

\section{Peak discharge rates}

The lower $\Delta \mathrm{F}$ values in the older adults were also accompanied by reduced motor neurone discharge rates. The PIC is an important modulator of discharge rate output [8,29] and the available monoamines, serotonin and noradrenaline, facilitate PICs to increase motor neuronal gain and alter the input-output relationship according to the required output [25,29,81-84]. PICs can amplify synaptic input by more than five-fold, and are thus a determinant mechanism influencing the capacity for motor neurones to achieve the necessary discharge rates to obtain very high muscle activation levels [10-12]. We have recently shown that increases in discharge rate during triangular-shaped contractions at different force levels were strongly associated with $\Delta \mathrm{F}$ increases, and thus PIC amplitudes, using a within-subject design [29]. Data from the current study reveal an important contribution of $\Delta \mathrm{F}$ to motor neurone discharge rates, in which $\Delta \mathrm{F}$ explained $53 \%$ of the variance in peak discharge rate. Motor unit discharge rates are not only modulated by PICs, but also depend on the ionotropic input they receive. Evidence in both animal $[85,86]$ and human models [87] suggests that the synaptic input onto the motor neurone decreases with ageing, which may result from increases in intracortical inhibition or reduced intracortical facilitation in older adults [8890]. Therefore, the lower peak discharge rates observed in older adults is a result of the lower PIC amplitudes and reduced synaptic input (descending drive and afferent feedback) received by the motor neurones.

\section{Strengths and limitations}

The main strength of our study was the use of two validated [8,25,31] and a widely used methods to estimate PIC strength in humans [8,25,29,36,38,44,91]; however, both methods have limitations that should be pointed out [26]. The pairwise method [8] allowed us to obtain several pairs of motor units, having a larger amount of test units per participant. On the other hand, this method present a higher variance as a result of under and overestimation of $\Delta \mathrm{Fs}$ as a consequence of adopting control units with varied recruitment thresholds [31]. Recently, Afsharipour et al., [31] proposed the use of a composite control unit to reduce the 
$561 \Delta \mathrm{F}$ variance present in the conventional paired motor unit analysis. However, this method 562 requires some additional assumptions, such as the overlay of three lower threshold motor 563 units with a similar discharge rate profile and with recruitment threshold below 3\%. When 564 following these assumptions, there was an important reduction of motor unit pairs, 565 particularly for soleus. Therefore, we initially adopted the pairwise method as it permitted 566 comparison between soleus and tibialis anterior $\Delta \mathrm{Fs}$. We also ran the composite method as 567 an additional analysis to examine whether reducing $\Delta \mathrm{F}$ variability and removing the influence 568 of the recruitment threshold on $\Delta \mathrm{F}$ obtained with the pairwise method would affect our main 569 outcomes. Using both methods allowed us to identify a large difference in $\Delta \mathrm{F}$ between young 570 and older adults, minimising the methodological limitations. Furthermore, it is worth mentioning that the assessors were not blinded to age group when visually inspecting and editing the motor units and future studies should confirm this with blinded assessors.

The lower recruitment threshold observed in the older adults may have been a result of bias of the decomposition algorithm because of the greater proportion of this type of motor units in this population. Therefore, caution should be taken when interpreting the differences in the recruitment threshold of motor units identified between these two heterogeneous groups. Indeed, our analytical approach included the variable recruitment threshold when modelling $\Delta \mathrm{F}$ aiming to control any potential confounding factor related to potential different physiological behaviours of distinct populations of motor units. and tibialis anterior motor units recruited at a low force level (20\% of peak torque); this is a commonly-used force target and is also similar to forces that might be expected in daily activities such as standing [39] and walking. However, older adults perform activities such as chair rise and both stair ascent and descent at a higher level of effort relative to their maximum capability [74] than young adults. Consequently, the $\Delta \mathrm{F}$ data obtained during low

586 force levels might not represent the motor neurone PIC behaviour at these daily activities requiring higher intensity contractions. Indeed, there is evidence that the function and structure of higher threshold motor neurones are more affected than lower threshold motor neurones [1]. This could hypothetically indicate a greater impairment in PICs during higher intensity contractions. Moreover, daily activities require the activation of different muscle groups. Motor neurones from distinct muscles depict different discharge behaviours during ageing [14]; thus, it is possible that $\Delta \mathrm{F}$ behaviour might also differ. Therefore, we recommend that future studies investigate the effect of ageing on $\Delta \mathrm{F}$ values from different 
muscles and at different contraction intensities, and its influence on possible impairments in physical function.

The cross-sectional design and the small group of tested individuals are additional limitations inherent to our study that should be mentioned. A cross-section study does not allow one to parse out causation per se. Longitudinal studies would involve repeated data collection from the same sample over several years to provide a better understanding of the effects of ageing on $\Delta F$. In addition, the small group of non-sarcopaenic older adults with normal mobility tested in the present study does not allow our results to be extrapolated to populations with different characteristics or health conditions, such as very old (>85 years old), sarcopaenic, frail, or those with neurological disorders. They may also not represent individuals who consistently perform high levels of physical activity. Having a broader spectrum of older adults with low to high physical function and force levels would allow a more adequate investigation of the relationship between $\Delta \mathrm{F}$ with these parameters.

\section{Conclusions}

The present study provides novel evidence of reduced intrinsic motor neurone excitability in a group of non-sarcopaenic older adults with normal mobility by estimating PIC amplitudes using the paired-motor unit analyses. Older adults had substantially lower $\Delta \mathrm{Fs}$, and presumably PIC amplitudes, in both soleus and tibialis anterior than young adults with comparable physical activity level. This would likely influence the capacity of older individuals to activate the muscles, thus requiring a greater descending drive from cortical areas and hence level of volitional effort, and greater number of recruited motor units to achieve the same force level (relative to maximum). We also identified a small contribution of $\Delta \mathrm{F}$ to the between-subject variability in peak discharge rates. The present findings contribute to our understanding of the effects of ageing on motor neurone excitability, which is a potential mechanism underpinning motor functional loss during ageing; this hypothesis should be explicitly tested in future studies. Two logical next steps are: 1) to examine the effect of ageing on monoaminergic projections onto the motor neurones and their relationship with the reduced PIC amplitude observed in the present study; 2) to investigate the association between $\Delta \mathrm{F}$ values for motor units in different muscles and the variance in performance on clinical tests of motor function.

\section{ADDITIONAL INFORMATION}

\section{Data and code availability}


628 The dataset and $\mathrm{R}$ code are available at https://github.com/orssatto/PICs-ageing.

629

\section{Competing interests}

631 The authors declare no competing interest related to this manuscript.

632

633

634

635 Author contributions

636 LBRO, AJS, AJB, and GST contributed with the conception and design of the work. LBRO

637 acquired data. RLS developed the MATLAB script for $\triangle \mathrm{F}$, peak discharge rate, and

638 recruitment threshold calculation. LBRO conducted the biological signals data analyses, and

639 DNB developed the R script and conducted statistical analyses. All authors interpreted and

640 discussed the data, drafted the manuscript, and revised it critically providing important

641 intellectual content.

642

643 All authors approved the final version of the manuscript; agree to be accountable for all

644 aspects of the work in ensuring that questions related to the accuracy or integrity of any part

645 of the work are appropriately investigated and resolved; and qualify for authorship, and all

646 those who qualify for authorship are listed.

647

$648 \quad$ Funding

649 There is no specific funding related to this manuscript. 


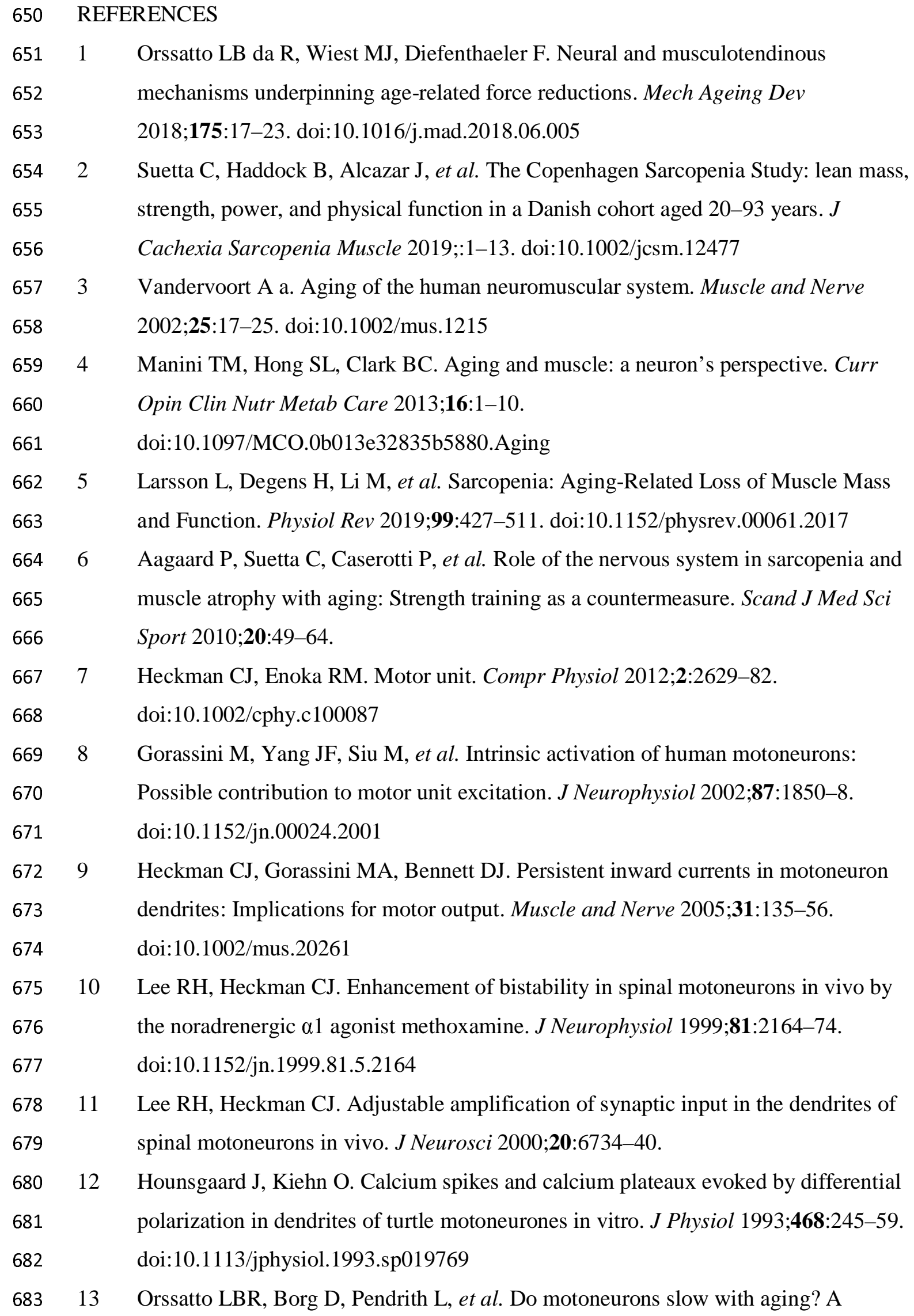


systematic review and meta-analysis with meta-regression. bioRxiv. 2020. doi:10.1101/2020.12.15.422812

14 Kirk EA, Christie AD, Knight CA, et al. Motor unit firing rates during constant isometric contraction $\square$ : establishing and comparing an age-related pattern among muscles. J Appl Physiol Published Online First: 2021. doi:https://doi.org/10.1152/japplphysiol.01047.2020

15 Christie A, Kamen G. Doublet discharges in motoneurons of young and older adults. $J$ Neurophysiol 2006;95:2787-95. doi:10.1152/jn.00685.2005

16 Piotrkiewicz M, Kudina L, Mierzejewska J, et al. Age-related change in duration of afterhyperpolarization of human motoneurones. J Physiol 2007;585:483-90. doi:10.1113/jphysiol.2007.142356

17 Morales FR, Boxer PA, Fung SJ, et al. Basic electrophysiological properties of spinal cord motoneurons during old age in the cat. J Neurophysiol 1987;58:180-94. doi:10.1152/jn.1987.58.1.180

18 Kalmar JM, Button DC, Gardiner K, et al. Caloric restriction does not offset ageassociated changes in the biophysical properties of motoneurons. J Neurophysiol 2009;101:548-57. doi:10.1152/jn.90617.2008

19 Liu KY, Kievit RA, Tsvetanov KA, et al. Noradrenergic-dependent functions are associated with age-related locus coeruleus signal intensity differences. Nat Commun 2020;11:1-9. doi:10.1038/s41467-020-15410-w

20 Shibata E, Sasaki M, Tohyama K, et al. Age-related changes in locus ceruleus on neuromelanin magnetic resonance imaging at 3 Tesla. Magn Reson Med Sci 2006;5:197-200. doi:10.2463/mrms.5.197

21 Ko ML, King MA, Gordon TL, et al. The effects of aging on spinal neurochemistry in the rat. Brain Res Bull 1997;42:95-8. doi:10.1016/S0361-9230(96)00216-X

22 Míguez JM, Aldegunde M, Paz-Valiñas L, et al. Selective changes in the contents of noradrenaline, dopamine and serotonin in rat brain areas during aging. J Neural Transm 1999;106:1089-98. doi:10.1007/s007020050225

23 Johnson H, Ulfhake B, Dagerlind A, et al. The serotoninergic bulbospinal system and brainstern $\square$ spinal cord content of serotonin $\square$, TRH $\square$, and substance P $\square$ like immunoreactivity in the aged rat with special reference to the spinal cord motor nucleus. Synapse 1993;15:63-89. doi:10.1002/syn.890150108

24 Latella C. Pick me, Pick me! Rationale for investigating persistent inward currents (PICs) and associated exercise effects in the ageing neuromuscular system. J Physiol 
2021;599:1957-9. doi:10.1113/JP281324

25 Powers RK, Nardelli P, Cope TC. Estimation of the contribution of intrinsic currents to motoneuron firing based on paired motoneuron discharge records in the decerebrate cat. J Neurophysiol 2008;100:292-303. doi:10.1152/jn.90296.2008

26 Stephenson JL, Maluf KS. Dependence of the paired motor unit analysis on motor unit discharge characteristics in the human tibialis anterior muscle. J Neurosci Methods 2011;198:84-92. doi:10.1016/j.jneumeth.2011.03.018

27 Holobar A, Zazula D. Multichannel blind source separation using convolution Kernel compensation. IEEE Trans Signal Process 2007;55:4487-96. doi:10.1109/TSP.2007.896108

28 Enoka RM. Physiological validation of the decomposition of surface EMG signals. $J$ Electromyogr Kinesiol 2019;46:70-83. doi:10.1016/j.jelekin.2019.03.010

29 Orssatto LBR, Mackay K, Shield AJ, et al. Estimates of persistent inward currents increase with the level of voluntary drive in low-threshold motor units of plantar flexor muscles. J Neurophysiol 2021;:6. doi:10.1152/jn.00697.2020

30 Revill AL, Fuglevand AJ. Effects of persistent inward currents, accommodation, and adaptation on motor unit behavior: A simulation study. J Neurophysiol 2011;106:1467-79. doi:10.1152/jn.00419.2011

31 Afsharipour B, Manzur N, Duchcherer J, et al. Estimation of self-sustained activity produced by persistent inward currents using firing rate profiles of multiple motor units in humans. J Neurophysiol 2020;124:63-85. doi:10.1152/jn.00194.2020

32 Polcyn AE, Kerrigan DC, Collins JJ. Age-Related Changes in the Initiation of Gait: Degradation of Central Mechanisms for Momentum Generation. Arch Phys Med Rehabil 1998;79:1582-9.

33 Laughton CA, Slavin M, Katdare K, et al. Aging, muscle activity, and balance control: Physiologic changes associated with balance impairment. Gait Posture 2003;18:101-8. doi:10.1016/S0966-6362(02)00200-X

34 Hagströmer M, Oja P, Sjöström M. The International Physical Activity Questionnaire (IPAQ): a study of concurrent and construct validity. Public Health Nutr 2006;9:75562. doi:10.1079/phn2005898

35 IPAQ. Guidelines for Data Processing and Analysis of the International Physical Activity Questionnaire (IPAQ) - Short and Long Forms. 2005. https://sites.google.com/site/theipaq/scoring-protocol

36 Trajano GS, Taylor JL, Orssatto LBR, et al. Passive muscle stretching reduces 
estimates of persistent inward current strength in soleus motor units. $J$ Exp Biol 2020;223:jeb.229922. doi:10.1242/jeb.229922

37 Kim EH, Wilson JM, Thompson CK, et al. Differences in Estimated Persistent Inward Currents Between Ankle Flexors and 2 Extensors in Humans. J Neurophysiol 2020;21:1-9.

38 Hassan AS, Thompson CK, Negro F, et al. Impact of parameter selection on estimates of motoneuron excitability using paired motor unit analysis. J Neural Eng 2020;17. doi:10.1088/1741-2552/ab5eda

39 Cattagni T, Scaglioni G, Laroche D, et al. The involvement of ankle muscles in maintaining balance in the upright posture is higher in elderly fallers. Exp Gerontol 2016;77:38-45. doi:10.1016/j.exger.2016.02.010

40 da Silva ME, Orssatto LB da R, Bezerra E de S, et al. Reducing measurement errors during functional capacity tests in elders. Aging Clin Exp Res 2018;30:595-603. doi:10.1007/s40520-017-0820-x

41 Bischoff HA, Stähelin HB, Monsch AU, et al. Identifying a cut-off point for normal mobility: A comparison of the timed 'up and go' test in community-dwelling and institutionalised elderly women. Age Ageing 2003;32:315-20. doi:10.1093/ageing/32.3.315

42 Holobar A, Minetto MA, Farina D. Accurate identification of motor unit discharge patterns from high-density surface EMG and validation with a novel signal-based performance metric. J Neural Eng 2014;11. doi:10.1088/1741-2560/11/1/016008

43 Binder MD, Powers RK, Heckman CJ. Nonlinear Input-Output Functions of Motoneurons. Physiology (Bethesda) 2020;35:31-9. doi:10.1152/physiol.00026.2019

44 Udina E, D’Amico J, Bergquist AJ, et al. Amphetamine increases persistent inward currents in human motoneurons estimated from paired motor-unit activity. $J$ Neurophysiol 2010;103:1295-303. doi:10.1152/jn.00734.2009

45 Vandenberk MS, Kalmar JM. An evaluation of paired motor unit estimates of persistent inward current in human motoneurons. J Neurophysiol 2014;111:1877-84. doi:10.1152/jn.00469.2013

46 Desmedt BJE, Godaux E. Ballistic contractions in man: Characteristic recruitment pattern of single motor units of the tibialis anterior muscle. J Physiol 1977;264:67393.

47 Kuznetsova A, Brockhoff PB, Christensen RHB. lmerTest Package: Tests in Linear Mixed Effects Models . J Stat Softw 2017;82. doi:10.18637/jss.v082.i13 
48 Lenth R V., Buerkner P, Herve M, et al. emmeans: Estimated Marginal Means, aka Least-Squares Means - R package. 2021.https://github.com/rvlenth/emmeans

49 Nakagawa S, Johnson PCD, Schielzeth H. The coefficient of determination R2 and intra-class correlation coefficient from generalized linear mixed-effects models revisited and expanded. J R Soc Interface 2017;14. doi:10.1098/rsif.2017.0213

50 Cruz-Jentoft AJ, Bahat G, Bauer J, et al. Sarcopenia: revised European consensus on definition and diagnosis. Age Ageing 2019;:1-16. doi:10.1093/ageing/afy169

51 Kamen G, Sullivan R, Rubinstein S, et al. Evidence of self-sustained motoneuron firing in young and older adults. J Electromyogr Kinesiol 2006;16:25-31. doi:10.1016/j.jelekin.2005.06.008

52 Harvey PJ, Li X, Li Y, et al. Endogenous monoamine receptor activation is essential for enabling persistent sodium currents and repetitive firing in rat spinal motoneurons. J Neurophysiol 2006;96:1171-86. doi:10.1152/jn.00341.2006

53 Cassano T, Gaetani S, Morgese MG, et al. Monoaminergic Changes in Locus Coeruleus and Dorsal Raphe Nucleus Following Noradrenaline Depletion. Neurochem Res 2009;34:1417-26. doi:10.1007/s11064-009-9928-5

54 Liu KY, Acosta-Cabronero J, Cardenas-Blanco A, et al. In vivo visualization of agerelated differences in the locus coeruleus. Neurobiol Aging 2019;74:101-11. doi:10.1016/j.neurobiolaging.2018.10.014

55 Michaud M, Balardy L, Moulis G, et al. Proinflammatory cytokines, aging, and agerelated diseases. J Am Med Dir Assoc 2013;14:877-82. doi:10.1016/j.jamda.2013.05.009

56 Pannese E. Morphological changes in nerve cells during normal aging. Brain Struct Funct 2011;216:85-9. doi:10.1007/s00429-011-0308-y

57 Nikoletopoulou V, Tavernarakis N. Calcium homeostasis in aging neurons. Front Genet 2012;3:1-17. doi:10.3389/fgene.2012.00200

58 Misgeld T. Lost in elimination: mechanisms of axonal loss. e-Neuroforum 2011;2:2134. doi:10.1007/s13295-011-0017-2

59 Selman C, Blount JD, Nussey DH, et al. Oxidative damage, ageing, and life-history evolution: Where now? Trends Ecol Evol 2012;27:570-7. doi:10.1016/j.tree.2012.06.006

60 Gordon T, Hegedus J, Tam SL. Adaptive and Maladaptive Motor Axonal Sprouting in Aging and Motoneuron Disease. Neurol Res 2004;26:174-85.

doi:10.1179/016164104225013806 
82061 Piasecki M, Ireland A, Jones DA, et al. Age-dependent motor unit remodelling in human limb muscles. Biogerontology 2016;17:485-96. doi:10.1007/s10522-015-96273

Dunia R, Buckwalter G, Defazio T, et al. Decreased duration of Ca2+-mediated plateau potentials in striatal neurons from aged rats. J Neurophysiol 1996;76:2353-63. doi:10.1152/jn.1996.76.4.2353

Di Iorio A, Cherubini A, Volpato S, et al. Markers of inflammation, Vitamin E and peripheral nervous system function. The InCHIANTI study. Neurobiol Aging 2006;27:1280-8. doi:10.1016/j.neurobiolaging.2005.07.004

Klass M, Baudry S, Duchateau J. Age-related decline in rate of torque development is accompanied by lower maximal motor unit discharge frequency during fast contractions. J Appl Physiol 2008;104:739-46. doi:10.1152/japplphysiol.00550.2007 Hyngstrom AS, Johnson MD, Miller JF, et al. Intrinsic electrical properties of spinal motoneurons vary with joint angle. Nat Neurosci 2007;10:363-9. doi:10.1038/nn1852 Hyngstrom AS, Johnson MD, Heckman CJ. Summation of excitatory and inhibitory synaptic inputs by motoneurons with highly active dendrites. J Neurophysiol 2008;99:1643-52. doi:10.1152/jn.01253.2007

Bui T V., Grande G, Rose PK. Relative location of inhibitory synapses and persistent inward currents determines the magnitude and mode of synaptic amplification in motoneurons. J Neurophysiol 2008;99:583-94. doi:10.1152/jn.00718.2007

8 Bui T V., Grande G, Rose PK. Multiple modes of amplification of synaptic inhibition to motoneurons by persistent inward currents. J Neurophysiol 2008;99:571-82. doi:10.1152/jn.00717.2007

Kuo JJ, Lee RH, Johnson MD, et al. Active Dendritic Integration of Inhibitory Synaptic Inputs in Vivo. J Neurophysiol 2003;90:3617-24. doi:10.1152/jn.00521.2003

0 Kido A, Tanaka N, Stein RB. Spinal excitation and inhibition decrease as humans age. Can J Physiol Pharmacol 2004;82:238-48. doi:10.1139/y04-017

7 Chalmers GR, Knutzen KM. Recurrent inhibition in the soleus motor pool of elderly and young adults. Electromyogr Clin Neurophysiol 2004;44:413-21.

2 Hounsgaard J, Hultborn H, Jespersen B, et al. Bistability of alpha $\square$ motoneurones in the decerebrate cat and in the acute spinal cat after intravenous $5 \square$ hydroxytryptophan. J Physiol 1988;405:345-67. doi:10.1113/jphysiol.1988.sp017336

73 Rozand V, Sundberg CW, Hunter SK, et al. Age-related Deficits in Voluntary Activation: A Systematic Review and Meta-analysis. Med Sci Sports Exerc 

2020;52:549-60. doi:10.1249/MSS.0000000000002179

74 Hortobágyi T, Mizelle C, Beam S, et al. Old adults perform activities of daily living near their maximal capabilities. J Gerontol A Biol Sci Med Sci 2003;58:M453-60. doi:10.1093/gerona/58.5.M453

75 Richardson CA, Glynn NW, Ferrucci LG, et al. Walking energetics, fatigability, and fatigue in older adults: The study of energy and aging pilot. Journals Gerontol - Ser A Biol Sci Med Sci 2015;70:487-94. doi:10.1093/gerona/glu146

76 Csapo R, Alegre LM. Effects of resistance training with moderate vs heavy loads on muscle mass and strength in the elderly: A meta-analysis. Scand J Med Sci Sports 2016;26:995-1006. doi:10.1111/sms.12536

77 da Rosa Orssatto LB, de la Rocha Freitas C, Shield AJ, et al. Effects of resistance training concentric velocity on older adults' functional capacity: A systematic review and meta-analysis of randomised trials. Exp. Gerontol. 2019;127. doi:10.1016/j.exger.2019.110731

78 Barry BK, Pascoe MA, Jesunathadas M, et al. Rate coding is compressed but variability is unaltered for motor units in a hand muscle of old adults. J Neurophysiol 2007;97:3206-18. doi:10.1152/jn.01280.2006

79 Enoka RM, Duchateau J. Rate coding and the control of muscle force. Cold Spring Harb Perspect Med 2017;7. doi:10.1101/cshperspect.a029702

80 Li Y, Gorassini MA, Bennett DJ. Role of Persistent Sodium and Calcium Currents in Motoneuron Firing and Spasticity in Chronic Spinal Rats. J Neurophysiol 2004;91:767-83. doi:10.1152/jn.00788.2003

81 Huh S, Siripuram R, Lee RH, et al. PICs in motoneurons do not scale with the size of the animal: A possible mechanism for faster speed of muscle contraction in smaller species. J Neurophysiol 2017;118:93-102. doi:10.1152/jn.00045.2017

82 Heckman CJ. Computer simulations of the effects of different synaptic input systems on the steady-state input-output structure of the motoneuron pool. J Neurophysiol 1994;71:1727-39. doi:10.1152/jn.1994.71.5.1727

83 Heckman CJ, Binder MD. Computer simulation of the steady-state input-output function of the cat medial gastrocnemius motoneuron pool. J Neurophysiol 1991;65:952-67. doi:10.1152/jn.1991.65.4.952

84 Powers RK, Heckman CJ. Contribution of intrinsic motoneuron properties to discharge hysteresis and its estimation based on paired motor unit recordings: A simulation study. J Neurophysiol 2015;114:184-98. doi:10.1152/jn.00019.2015 
85 Maxwell N, Castro RW, Sutherland NM, et al. $\alpha$-Motor neurons are spared from aging while their synaptic inputs degenerate in monkeys and mice. Aging Cell 2018;17. doi:10.1111/acel.12726

86 Chase MH, Morales FR, Boxer PA, et al. Aging of motoneurons and synaptic processes in the cat. Exp Neurol 1985;90:471-8. doi:10.1016/0014-4886(85)90035-4

87 Rowe JB, Siebner H, Filipovic SR, et al. Aging is associated with contrasting changes in local and distant cortical connectivity in the human motor system. Neuroimage 2006;32:747-60. doi:10.1016/j.neuroimage.2006.03.061

88 Todd G, Taylor JL, Gandevia SC. Measurement of voluntary activation of fresh and fatigued human muscles using transcranial magnetic stimulation. $J$ Physiol 2003;551:661-71. doi:10.1113/jphysiol.2003.044099

89 Opie GM, Hand BJ, Semmler JG. Age-related changes in late synaptic inputs to corticospinal neurons and their functional significance: A paired-pulse TMS study. Brain Stimul 2020;13:239-46. doi:10.1016/j.brs.2019.08.003

90 McGinley M, Hoffman RL, Russ DW, et al. Older adults exhibit more intracortical inhibition and less intracortical facilitation than young adults. Exp Gerontol 2010;45:671-8. doi:10.1016/j.exger.2010.04.005

91 Hassan AS, Kim EH, Khurram OU, et al. Properties of Motor Units of Elbow and Ankle Muscles Decomposed Using High-Density Surface EMG. Proc Annu Int Conf IEEE Eng Med Biol Soc EMBS 2019;:3874-8. doi:10.1109/EMBC.2019.8857475 